\title{
Dark Matter Annihilation in the light of EGRET, HEAT, WMAP, INTEGRAL and ROSAT
}

\author{
Iris Gebauer* \\ Institut für Experimentelle Kernphysik, Universiät Karlsruhe \\ E-mail: gebauer@ekp.uni-karlsruhe.de
}

The ROSAT Galactic wind observations confirm that our Galaxy launches supernova (SN) driven Galactic winds with wind speeds of about $150 \mathrm{~km} / \mathrm{s}$ in the Galactic plane. Galactic winds of this strength are incompatible with current isotropic models for Cosmic Ray (CR) transport as implemented in the GALPROP code.

In order to reproduce our local CRs in the presence of Galactic winds, charged CRs are required to be much more localized than in the standard isotropic GALPROP models. This requires that anisotropic diffusion is the dominant diffusion mode in the interstellar medium (ISM), particularly that the diffusion in the disk and in the halo are different. In addition small scale phenomena such as trapping by molecular cloud complexes and the structure of our local environment (the local bubble or the local fluff) might influence the secondary CR production rate and our local CR density gradients.

We introduce an anisotropic convection driven transport model (aCDM) which is consistent with the Galactic wind observations by ROSAT. This automatically explains the large bulge/disk ratio as observed by INTEGRAL. Furthermore such models predict an increase in the $e^{+} /\left(e^{+}+e^{-}\right)$fraction as observed by PAMELA and HEAT, if the synchrotron constraints in the $408 \mathrm{MHz}$ and WMAP range are taken into account. This increase originates entirely from the transport properties of electrons and positrons, no additional contribution from Dark Matter (DM) is required. The aCDM is able to explain the absence of a positron annihilation signal from molecular clouds (MCs) as observed by INTEGRAL by virtue of a mechanism which confines and isotropizes CRs between MCs. Unlike isotropic models, this model does not rely on a flattened source distribution or a strong increase in the $X_{C O}$ scaling factor, but uses the supernova remnant (SNR) distribution as source distribution for CRs and a flat $X_{C O}$ scaling factor. We find that the EGRET excess of diffuse $\gamma$-rays currently cannot be explained by astrophysical effects in this type of model and that the interpretation of the EGRET excess as Dark Matter annihilation (DMA) is perfectly consistent with all observational constraints from local CR fluxes and synchrotron radiation.

Identification of dark matter 2008

August 18-22, 2008

Stockholm, Sweden

* Speaker. 


\section{Introduction}

Current CR transport models as implemented in the GALPROP code $^{1}$ are able to either explain our local charged CRs (conventional models) or the diffuse Galactic $\gamma$-rays (optimized models). The reason for this is that the locally observed proton spectrum is incompatible with the observed $\gamma$-rays by both, spectral shape and absolute flux. In isotropic diffusion dominated transport models this inconsistency cannot be explained by transport effects, because our local CRs are strongly linked to the interstellar CR population. As a possible explanation an additional component from DMA has been proposed (1). However, in this case isotropic transport models predict too many local antiprotons (2). It is shown that anisotropic diffusion models are constistent with the DMA interpretation of the EGRET excess.

\section{Anisotropic Convection Driven Transport Models}

The EGRET excess is not the only challenge for Galactic transport models. Self-consistent Galactic wind calculations have led to the conclusion that convective transport plays a non-negligible rôle in our Galaxy. A recent analysis of the ROSAT X-ray data has confirmed that our Galaxy indeed launches winds with speeds of $150 \mathrm{~km} / \mathrm{s}$ in the plane at the position of the sun and up to 800 $\mathrm{km} / \mathrm{s}$ in the halo (3). Although this is a comparably low speed (starburst Galaxies launch winds with speeds up to $3000 \mathrm{~km} / \mathrm{s}$ ), the impact upon CR transport is significant, because convective transport occurs only in the direction perpendicular to the plane. As expected, the wind velocities appear to be roughly proportional to the SNR distribution. Transport models with isotropic diffusion can allow for convection velocity gradients of only $10 \mathrm{~km} / \mathrm{s} / \mathrm{kpc}$ (with zero wind speed at $\mathrm{z}=0$ $\mathrm{kpc}$ ), since for larger velocities the constraints from radioactive isotopes and secondary particle production cannot be met (4). To account for the observed wind velocities anisotropic diffusion modes have to be considered. Convection velocities compatible with the ROSAT observations significantly reduce the distance CRs travel in our Galaxy before they enter the convection zone and escape, thus leading to a model which allows for both - an effective decoupling of local CRs from the global CR population as required by an astrophysical explanation of the EGRET excess and a small collection volume for all CR species including antiprotons from DMA. Such a model not only decouples the local CR spectra from the Galactic average, it also explains the large bulge/disk ratio as observed in the $511 \mathrm{keV}$ line by INTEGRAL (5) purely by the properties of CR transport. The basic observation is the high intensity from the region of the Galactic bulge and a rather low signal from the disk, although the opposite is expected (6). The annihilation signal in the disk can be entirely explained by the decay of ${ }^{26} \mathrm{Al}$ from core collapse SNs, leaving no room for positrons from SNIa. Isotropic transport models have to invoke additional sources which are confined to the bulge in order to partially (7) or entirely (8) explain the observed signal from the bulge, but they cannot explain why there is no annihilation signal from SNIa in the disk. In an aCDM positrons from SNIa are produced predominantly in regions with strong Galactic winds and so the $\mathrm{MeV}$ positrons from SNIa are almost immediately blown into the halo. In the bulge, where the lower SN rate and the strong gravitational potential reduce the wind strength, positrons have enough time to annihilate.

\footnotetext{
${ }^{1}$ The GALPROP code is publicly available from http://galprop.stanford.edu
} 
A second surprising result from INTEGRAL is the observation that positrons do not annihilate in molecular clouds (MCs). A spectral analysis of the annihilation line indicates that practically all annihilation takes place in the atomic component of the gas, although molecular clouds have an average density of more than half of the gas (9). One could argue that the volume filling factor of molecular clouds is too small to be found by positrons. However, the high magnetic field inside MCs appears to correlate with the interstellar magnetic field (10). In such a model MCs will act as magnetic mirrors and positrons are likely to be reflected and confined between MCs (11).

Note, that Galactic winds in agreement with ROSAT are also able to explain the observed soft gradient in diffuse $\gamma$-rays without invoking a flattened source distribution or an increase in the $X_{\mathrm{CO}^{-}}$ scaling factor: the peak in the gas distribution coincides with the peak in the SNR distribution, consequently the $\mathrm{CR}$ interaction rate in this region, which leads to a relative overproduction of diffuse $\gamma$-rays in the isotropic models, is reduced in an aCDM.

INTEGRAL and ROSAT form strong constraints for any transport model. We have implemented the transport picture described above into the publicly available GALPROP code. The main features of our implementation are: i) fast propagation perpendicular to the disk by turbulent diffusion and convection in agreement with the ROSAT X-ray data ii) slow diffusion in the disk and a possible trapping mechanism by molecular clouds which would increase the CR interaction rate in the atomic component of the gas. In addition we allow for local variations in all transport parameters in order to account for our local Galactic environment. Compared to isotropic models CRs travel smaller distances in an aCDM leading to a stronger impact of local transport phenomena.

We tune the transport parameters for local CR spectra and allow for an additional contribution from DMA in order to describe both, the diffuse $\gamma$-rays and local CR spectra (conventional aCDM). Local protons, electrons, $B / C$-fraction, ${ }^{10} \mathrm{Be} /{ }^{9} \mathrm{Be}$-fraction, $e^{+} /\left(e^{+}+e^{-}\right)$-fraction, diffuse $\gamma$-rays and the $22 \mathrm{GHz}$ synchrotron profile for the haze region are shown in figures 1 to 3 . A breakless proton injection spectrum and a breakless diffusion coefficient turn out to be sufficient to describe the locally observed CR fluxes and the diffuse $\gamma$-rays. Note, that in this model the increase in $e^{+} /\left(e^{+}+e^{-}\right)$-fraction, as observed by PAMELA, is a natural consequence of CR transport: the breaks in $e^{+} /\left(e^{+}+e^{-}\right)$-fraction are mainly determined by the electron and proton injection spectra, momentum losses and gains only play a minor rôle. In a conventional aCDM this naturally yields a positron spectrum which is slightly harder than the electron spectrum and consequently gives rise to the observed increase in $e^{+} /\left(e^{+}+e^{-}\right)$-fraction (this interpretation is strongly supported by the proton and electron measuments from AMS up to $30 \mathrm{GeV}$, which show an even steeper increase in the $p /\left(p+e^{-}\right)$-fraction). Compared to isotropic models the contribution from DMA in local CRs is small, since most of the positrons and antiprotons from DMA are produced in the halo, thus drifting away by convection. Note, that in an aCDM no additional fine-tuning is required to describe the data on local antiprotons: convection speeds are fixed by the ROSAT observations and transport parameters are fixed by local CR spectra and constraints from synchrotron radiation. The right side of figure 3 shows the latitude profile of the synchrotron radiation in the so-called WMAP haze region for $22 \mathrm{GHz}$. The WMAP haze consists of an excess of microwave emission from a small region close to the Galactic center. It has been suggested that this signal could be synchrotron emission from relativistic electrons and positrons, possibly originating from DMA in a cuspy halo (13). However, even in a cored profile the synchrotron radiation from the disk shows a steep increase as shown in figure 3 , while the intensity of synchrotron radiation from DMA (as 

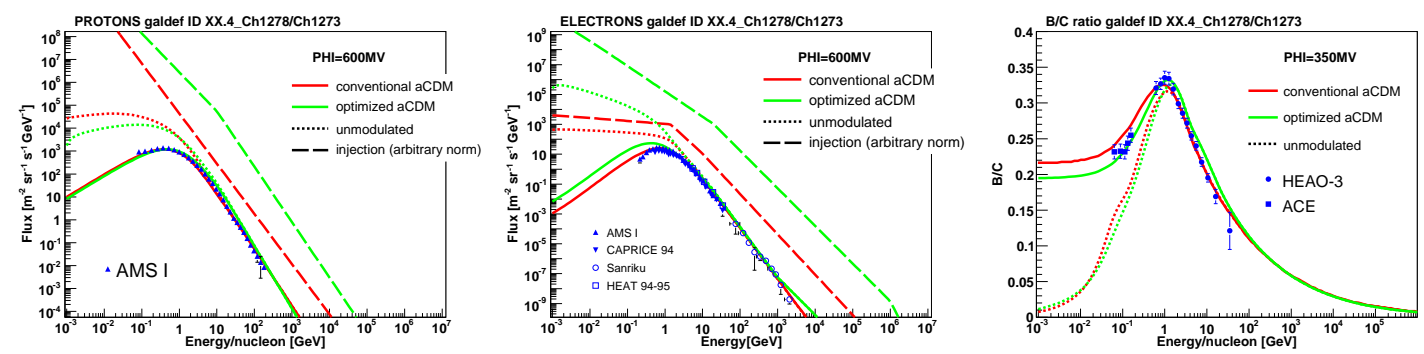

Figure 1: Left: Local proton flux for a conventional (red) and optimized aCDM (green). The full lines correspond to local modulated fluxes, the dotted lines are local unmodulated spectra and the dashed lines are the injection spectra. Center: Local electron spectrum, line coding as for protons. Right: Local $B / C$ ratio in an aCDM, line coding as for protons.
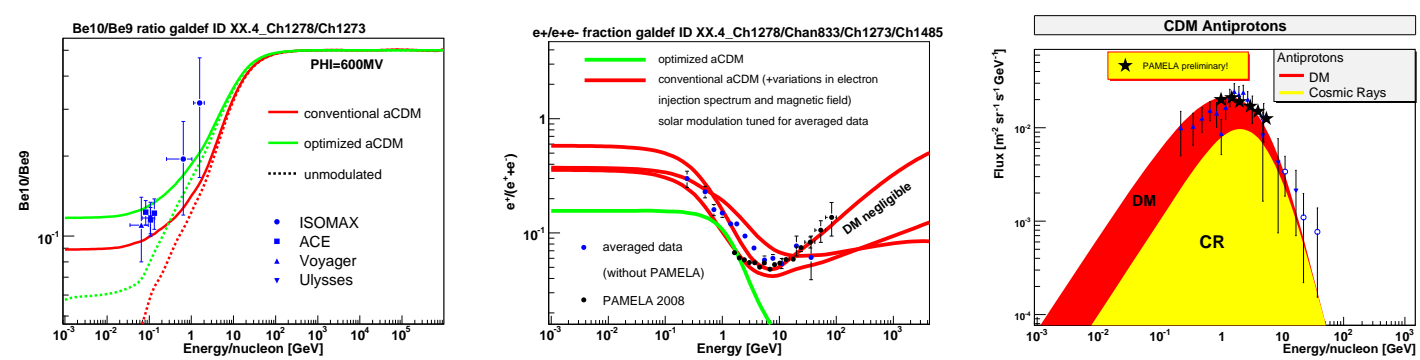

Figure 2: Left: Local ${ }^{10} \mathrm{Be} /{ }^{9} \mathrm{Be}$-fraction for an aCDM, line coding as for protons in figure 1. Center: Local $e^{+} / e^{+}+e^{-}$-fraction for an optimized aCDM (green) and a conventional aCDM (red). For the conventional aCDM the different red lines correspond to different combinations of electron injection spectra and magnetic fields, which are correlated by a fit to the synchrotron radiation spectra of the Haslam skymap and WMAP data. The averaged data are taken from (14) and do not include the PAMELA results. Right: Local antiprotons in a conventional aCDM. The contribution from CRs is very similar to the predictions of the isotropic models, while the contribution from DMA is reduced by virtue of the smaller collection volume.

required to explain the EGRET excess) is much lower. In order to check for astrophysical sources of the EGRET excess we tune the transport parameters with respect to diffuse $\gamma$-rays and try to reproduce our local charged CRs by variations of our local transport parameters (optimized aCDM). Figures 1 to 3 show the local protons, electrons, $B / C$-fraction, ${ }^{10} B e /{ }^{9} B e$-fraction, $e^{+} /\left(e^{+}+e^{-}\right)$fraction and diffuse $\gamma$-rays in an optimized aCDM. As in the isotropic model the $\gamma$-rays require a break in the proton injection spectrum at $10 \mathrm{GeV}$ which is not expected from the acceleration of protons by SN explosions (12). Unlike the isotropic models, in this type of model we are able to describe the observed spectra of protons and nuclei reasonably well by choosing our local transport parameters accordingly. Still, in an optimized aCDM local electrons seem to be incompatible with the locally observed proton spectrum as can be seen from the $e^{+} /\left(e^{+}+e^{-}\right)$-fraction in figure 2 .

\section{Conclusion}

We have presented a transport model for Galactic CRs compatible with the wind velocities observed by ROSAT. In such a model the INTEGRAL $511 \mathrm{keV}$ line and its spectral morphology, the synchrotron radiation observed by WMAP and the locally observed positron and electron spec- 

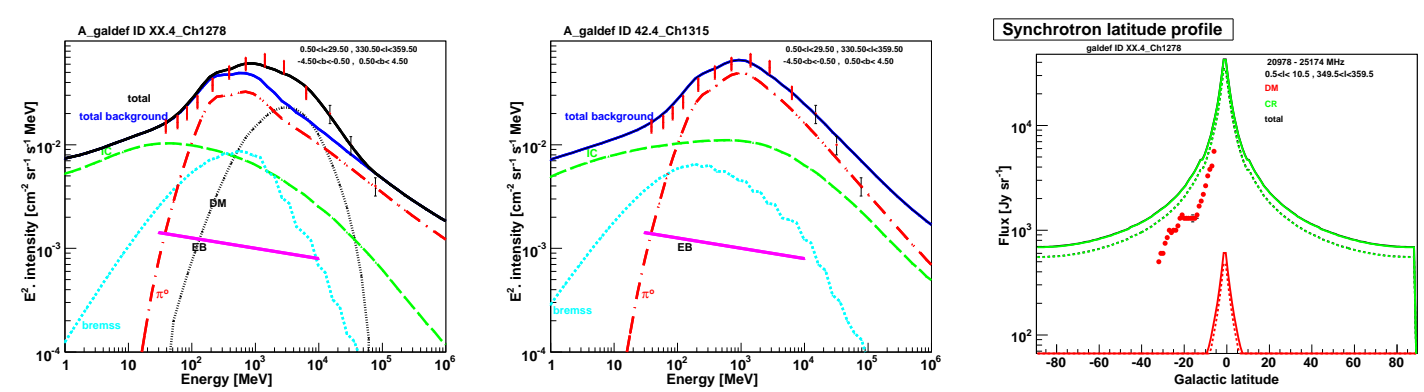

Figure 3: Diffuse $\gamma$-rays from the Galactic center region in a conventional (left) and optimized (center) aCDM. Right: Latitude distribution of the synchrotron radiation in the small longitude range where the haze has been measured. The top solid curve is the total flux, the red dots represent the haze and the lower curve is the contribution from the DMA interpretation of the EGRET excess.

tra are explained entirely as a consequence of CR transport. However, without DMA this type of model is currently not able to explain the diffuse $\gamma$-rays as observed by EGRET by astrophysical effects in concordance with the local CR spectra. We showed that the DMA interpretation of the EGRET excess is compatible with the constraints from CR transport.

\section{References}

[1] W. de Boer, C. Sander, V. Zhukov, A. V. Gladyshev and D. I. Kazakov, A\&A 44451 (2005).

[2] L. Bergström et al., JCAP 0605006 (2006).

[3] D. Breitschwerdt, Nature 452, 7189826 (2008) and references therein.

[4] A. W. Strong, I. V. Moskalenko and V. S. Ptuskin, Ann. Rev. Nucl. Part. Sci. 57285 (2007).

[5] J. Knödlseder et al., A\&A 441513 (2005).

[6] N. Prantzos, N. A\&A 449869 (2005) and [astro-ph/0809.2491].

[7] G. Weidenspointner et al., Nature 451159 (2008).

[8] C. Boehm, D. Hooper, J. Silk, M. Casse, and J. Paul, Phys. Rev. Lett. 92101301 (2004).

[9] P. Jean et al. Astron. Astrophys. 445579 (2005) 579.

[10] J. L. Han and J. S. Zhang, astro-ph/0611213.

[11] B.D.G. Chandran, Space Sci. Rev. 99271 (2000).

[12] V. L. Ginzburg, V. A. Dogiel, V. S. Berezinsky, S. V. Bulanov and V. S. Ptuskin, Astrophysics of cosmic rays, Amsterdam, Netherlands: North-Holland (1990) $534 \mathrm{p}$

[13] D. P. Finkbeiner, Astrophys. J. 614186 (2004) and Phys. Rev. D 76083012 (2007).

[14] C. H. Chung, H. Gast, J. Olzem and S. Schael, The anomaly in the cosmic-ray positron spectrum, in Proc. of SUSY07, July 26 - August 1, 2007 Karlsruhe, Germany. 